\title{
LA ESTRATEGIA DE BRASIL EN LA ÉPOCA DE LA GLOBALIZACIÓN ECONÓMICA
}

\author{
BRAZILIAN STRATEGY IN THE AGE OF ECONOMIC GLOBALIZATION
}

Alex Schnake Gálvez ${ }^{1}$

Fecha de Recepción:28/09/2011

Fecha de Aceptación27/04/2012

\section{RESUMEN}

El fenómeno de la globalización económica y sus principales características, modelo neoliberal e intensificación del comercio internacional, ha marcado las tendencias de la economía mundial durante las últimas décadas. Por su parte, América Latina no ha estado al margen de este proceso. En este artículo analizaremos cómo la globalización de los mercados ha influido en la política económica de Brasil, principal motor económico de la región. Adicionalmente, veremos cómo la economía brasileña ha logrado una exitosa adaptación a las nuevas tendencias. En efecto, revisaremos cómo Brasil ha acelerando sus tasas de crecimiento, pero a la vez, ha conseguido una distribución cada vez más equitativa de los beneficios que la misma globalización promete.

Palabras claveBrasil, globalización, política económica, comercio internacional.

\begin{abstract}
The phenomenon of the economic globalization -and its main features, neoliberalism and international trade- has determined the global economic trends during the last decades. Latin America has not been excluded from this process. We will analyze how the globalization of markets has influenced on the Brazilian economic policy which is the leading economic country in the region. In addition, we will be able to appreciate how the Brazilian economy has achieved a successful adaptation to the new tendencies. Actually, we will review how Brazil has accelerated its growth rates but at the same time obtained an increasingly fair distribution of the benefi ts promised by (globalization).
\end{abstract}

Key words:Brazil, globalization, economic policy, international trade.

\footnotetext{
1 Abogado. Ayudante en la Facultad de Derecho de la Universidad de Chile. Diplomado en Relaciones Internacionales, Globalización e Integración Regional de la Universidad Alberto Hurtado. Santiago, Chile. E.-mail: schnake.alex@gmail.com
}

doi: 10.7770/RCHDYCP-V3N1-ART344 


\section{Introducción}

El fenómeno de la globalización, en su sentido económico, ha sido probablemente el cambio más relevante que ha experimentado la economía y el comercio mundial durante los últimos 50 años. Las reformas económicas neoliberales Ilevadas a cabo por los gobiernos de Ronald Reagan en Estados Unidos y Margaret Thatcher en Gran Bretaña, fueron la punta de lanza de un proceso que se consolidaría a fines de los 80 con el Ilamado Consenso de Washington.

Durante las últimas décadas, de la mano de la llamada revolución de las comunicaciones, dicho proceso se ha ido profundizando. A la globalización económica o comercial, caracterizada por la liberalización y apertura de los mercados, se ha sumado la llamada globalización financiera, donde los actores principales son los mercados de valores o bolsas de comercio. En la actualidad, en estos mercados se transan miles de millones de dólares en cuestión de minutos, determinando en gran medida los rumbos de la economía global y la estabilidad de la misma.

En paralelo a este proceso, han aparecido nuevos jugadores o actores económicos globales, de características no institucionales. Se trata de las compañías transnacionales. Dichos actores han adquirido tal poder sobre el mercado internacional, que han desafiado continuamente la capacidad regulatoria de los Estados Nacionales, que, en términos generales, han visto disminuido su control sobre la economía.

Todos estos acontecimientos, para bien o para mal, han afectado a las economías latinoamericanas, las cuales se han adaptado en mejor o peor medida al nuevo escenario.

En un comienzo, uno de los efectos regionales más negativos del nuevo orden económico, fue la estimulación de un excesivo endeudamiento de las economías nacionales. Este proceso estuvo asociado a la abundancia de los denominados "petrodólares" administrados por la banca privada transnacional y que provocó que la década de los ochenta incluyera la peor recesión regional registrada desde la crisis de los años treinta ${ }^{2}$.

Por su parte, entre las experiencias positivas, se suele señalar el fenómeno chileno, país que durante la década de los 80 inició una progresiva rebaja de sus aranceles, abriéndose a los mercados norteamericano, europeo y también a los principales mercados asiáticos. Esta apertura, sumada a una legislación que estimuló la inversión extranjera, se tradujo en una rápida diversificación de sus mercados de exportación,

2 Di Filippo, Armando, Economía Política Global, [on line], [consultado el 14 de septiembre de 2011], "Disponible en la World Wide Web: http://www.difilippo.cl/descargar_ap.php?id=178", p.178. 
un mantenido aumento del crecimiento económico - medido en términos del PIB-y un reconocimiento mundial de país estable y confiable para los inversionistas. No obstante, dicha estrategia, también aumentó la dependencia económica chilena respecto de la exportación de sus productos primarios, disminuyó los incentivos para el desarrollo de una industria nacional y permitió que el país fuese extremadamente vulnerable a las crisis financieras de los mercados mundiales. En gran medida, esta vulnerabilidad explica la etapa de bajo crecimiento en que entró la economía chilena con posterioridad a la crisis asiática.

No obstante, lo singular del caso chileno, es que la etapa política en que se introdujeron las reformas liberales, así como la dimensión de su mercado, no permiten extrapolar sus efectos más allá de sus fronteras. Por lo mismo, y pensando en un mayor alcance de las conclusiones de este análisis, revisaremos cómo la globalización económica y sus dinámicas características -modelo económico neoliberal e intensificación del comercio internacional- han influido en las políticas del principal mercado de la región, Brasil.

\section{El poder económico de Brasil. Algunos antecedentes}

Antes de comenzar nuestro análisis es importante tener en cuenta la capacidad y condiciones económicas de base del Ilamado gigante del sur.

En primer término, Brasil siempre ha poseído una serie de condiciones connaturales para convertirse en potencia económica global. Según el último Censo realizado en 2010, en Brasil habitan 190.732.694 millones de personas ${ }^{3}$-más de un tercio de la población latinoamericana es brasileña-, por tanto, posee un potencial demográfico considerable. Por otro lado, en materia geográfica, Brasil cuenta con una superficie de 8,5 millones de $\mathrm{km}^{2}$, siendo el quinto país más grande del mundo. Brasil tiene una extensión territorial mayor que Australia e India -los que le siguen en el rankingy solo es superado por China, Estados Unidos, Canadá y Rusia. En consecuencia, solo por tamaño, la economía brasileña tiene extraordinarias condiciones.

En segundo lugar, Brasil es un país indudablemente rico en recursos naturales. Además de contar con la Selva Amazónica -el Ilamado pulmón verde del mundo-, Brasil posee importantes recursos energéticos. Además de biocombustibles, Brasil cuenta con grandes reservas petrolíferas. Asimismo, su capacidad agroalimentaria le ha permitido erigirse como uno de los grandes abastecedores mundiales en esta materia.

Brasil.gov.br, [on line], [consultado el 27 de abril de 2012]. "Disponible en la World Wide Web: http:// www.brasil.gov.br/sobre/brasil-1/brasil-en-numeros/demografia". 
Finalmente, en términos comerciales, Brasil es claramente el líder de la región, poseyendo una participación de alrededor de un tercio del PIB regional. Llevado a escala global, según los datos del Fondo Monetario Internacional, Brasil es actualmente la séptima economía del mundo, con un producto interno bruto (PIB) a valor de paridad de poder adquisitivo (PPA) de 2.393,954 billones de dólares ${ }^{4}$.

Sin embargo, a pesar de sus extraordinarias condiciones, la economía brasileña arrastra graves problemas que le impiden desarrollarse plenamente. Algunos de ellos son su elevada deuda pública -que llegó al 49,5\% del PIB el año 2006-, su alta dependencia en la exportación de materias primas (a pesar de contar con la industria más desarrollada de la región) y su alto nivel de desigualdad social (Brasil ocupa el lugar $73^{\circ}$ en el Índice de Desarrollo Humano ajustado por la Desigualdad desarrollado por el Programa de las Naciones Unidas para el Desarrollo, encontrándose en esta materia al nivel de países como Irán, Ucrania o Bosnia y Herzegovina ${ }^{5}$ ). Esto último, se ha mencionado como causa basal de una serie de problemas domésticos relacionados con la seguridad ciudadana, el narcotráfico y la criminalidad.

De este modo Brasil, si bien posee las condiciones para convertirse en un país desarrollado y una potencia económica global, pareciera aún contar con una serie de obstáculos que le impiden el salto al desarrollo.

Ahora bien, en el balance general, las últimas décadas dejan un claro saldo positivo. Luego de las reformas económicas neoliberales implementadas durante el gobierno de Fernando Henrique Cardoso, Brasil inició un ciclo de prosperidad -a pesar de contar con tasas de crecimiento relativamente bajas- que durante los últimos años el presidente Lula da Silva supo aprovechar y capitalizar.

Es reconocido que durante los gobiernos de Lula da Silva se implementaron una serie de políticas públicas bien diseñadas que permitieron incorporar a buena parte de la población brasileña a la creciente clase media. Esto significó integrar a la cadena de consumo a varios millones de brasileños que hasta esa época vivían bajo la línea de la pobreza. Asimismo, durante los mandatos de Lula, Brasil comenzó a erigirse como actor político y económico de talla mundial. Además de la formación de una serie de alianzas estratégicas y comerciales con la mayoría de las potencias emergentes (como los Ilamados $\mathrm{BRIC}^{6}$ o el IBSA ${ }^{7}$ ), Brasil demostró su liderazgo políti-

4 World Economic Outlook Database, April 2012. [on line], [consultado el 27 de abril de 2012]. “Disponible en la World Wide Web: http://www.imf.org/external/pubs/ft/weo/2012/01/weodata/index.aspx".

5 Informe sobre Desarrollo Humano 2010, Programa de las Naciones Unidas para el Desarrollo (PNUD), [on line], [consultado el 27 de abril de 2012]. "Disponible en la World Wide Web: http://hdr.undp.org/ en/media/HDR_2010_ES_Complete_reprint.pdf".

6 La sigla BRIC hace referencia a Brasil, Rusia, India y China.

7 La sigla IBSA hace referencia al Foro de Diálogo instalado en junio de 2003 entre India, Brasil y Sudáfrica. 
co global cuando en el marco del rechazo a las negociaciones de la Ronda de Doha -reunión de la Organización Mundial de Comercio (OMC) en Qatar, 2003- promovió la conformación del Ilamado G-20.

El balance positivo en la agenda política y económica brasileña de los últimos años consideramos se ha debido, en buena medida, a la capacidad de sus líderes para comprender las ventajas y desventajas del modelo que impone la globalización. En efecto, como explicaremos a continuación, Brasil ha sido capaz de administrar con éxito y hasta cierto punto corregir el modelo económico neoliberal. Asimismo, Brasil ha sido tremendamente eficaz al momento de aprovechar las oportunidades de comercio internacional que la globalización propicia. Y como veremos, el resultado ha sido una acelerada tasa de crecimiento económico con mayores componentes de equidad y una respetada posición en las esferas del comercio internacional.

\section{La evolución del modelo: del neoliberalismo al crecimiento con equidad}

En términos generales, los principales rasgos de la economía brasileña de fines de los 80 era contar con una altísima inflación y un modelo económico principalmente proteccionista. El proteccionismo brasileño comenzó a revertirse entre 1987 y 1988 cuando "se inició un proceso de reformulación de la política comercial, que incluyó modificaciones significativas en relación con diversas barreras arancelarias, entre otras: la flexibilización de las condiciones de financiamiento externo requeridas para la importación de bienes de capital, la reducción del número de artículos de importación prohibida, la administración más flexible de los programas anuales de importación que las empresas interesadas deben presentar" ${ }^{\prime \prime}$. Este proceso sería luego profundizado por las siguientes administraciones, en especial, por la de Fernando Henrique Cardoso quien introduciría reformas estructurales a la economía del país.

Sin embargo, el problema de la inflación tardaría más tiempo en ser modificado. Desde 1985, primer año del gobierno de José Sarney, Brasil experimentaba una galopante inflación. Esta llegaría hacia el año 1994 a la exorbitante cifra de 2.312\%. El punto de quiebre llegaría ese mismo año y lo marcaría el plan económico liderado por Fernando Henrique Cardoso, cuando este oficiaba de ministro de Hacienda de Itamar Franco. El Plan consistiría en una serie de medidas monetarias, donde destacó la introducción de la nueva moneda, el real. Al año siguiente, la inflación fue reducida al $28 \%$. El exitoso control de la inflación catapultaría a Cardoso a la presidencia brasileña, lugar desde el cual Ilevaría a cabo una serie de transformaciones al modelo económico, alineando a Brasil con la tendencia mundial de apertura al comercio exterior, privatizaciones y reformas al mercado del trabajo.

8 CEPAL, Transformación productiva con equidad. La tarea prioritaria del desarrollo de América Latina y el Caribe en los años noventa, Publicación de las Naciones Unidas, Santiago de Chile, 1990, № 33, p. 88. 
En términos concretos, y simplificando al máximo, los logros económicos de los periodos presidenciales de Cardoso resultarían ser la estabilización de la moneda, la disminución del gasto fiscal y el control de la deuda externa. No obstante, este capítulo de la historia política brasileña terminaría siendo una especie de periodo refundacional, basado en dos pilares: un sistema democrático estable y una política económica de corte liberal.

Es en este contexto que, en el año 2003, Lula da Silva asume el poder en Brasil. En un principio se creía que el nuevo presidente -de origen sindicalista y proveniente del ala izquierda brasileña- viraría el timón hacia un modelo económico de características socialistas, mediante la introducción de nuevas reformas estructurales. Sin embargo, nada de eso ocurrió. Si bien Da Silva aspiraba a una sociedad más igualitaria y a una mejor distribución de la riqueza, tenía el pragmatismo suficiente para comprender que la sociedad brasileña había cambiado. Para él, sus reformas solo podrían tener éxito si lograban "dialogar" -y en cierto sentido "superar"- con las reglas del juego económico capitalista. Y por lo mismo, apenas comenzó su gobierno, Lula da Silva estableció como prioridad la mantención de la estabilidad monetaria. Para ello, entre otras cosas, se propuso mantener en superávit las cuentas fiscales. Este hecho le significaría la confianza de la clase empresarial y de los principales prestamistas estatales -en particular, del Fondo Monetario Internacional- que miraban con recelo su llegada al poder.

No obstante, Lula no se dedicó simplemente a administrar las reformas liberales heredadas de Fernando Henrique Cardoso. El presidente sindicalista se enfocó en aprobar una serie de medidas de corte evidentemente progresista.

En primer lugar, decidió detener el proceso de privatizaciones de empresas públicas, eso sí, sin que ello significara regresar al modelo de empresa estatal de los años 60. Una de las claves de Lula fue introducir el concepto de las asociaciones público-privadas, figura mediante las cuales se enfrentarían las futuras obras de infraestructura que emprendiera el Estado. En este modelo asociativo "el Estado define qué hacer, conservando el papel dirigente, y realiza parte de las inversiones. Una vez finalizada la obra, las empresas privadas se convierten en explotadoras de los servicios (caminos, ferrocarriles, metro) con una rentabilidad mínima garantizada por un fondo público" ${ }^{\prime \prime}$. Estos proyectos de asociación fueron acompañados de proyectos de inversión y rescate de las principales empresas públicas que no habían sido privatizadas, además de medidas para mejorar la competitividad de las empresas de origen local.

9 Sallum Jr., Brasilio, La especificidad del gobierno de Lula. Hegemonía liberal, desarrollismo y populismo / en / Revista Nueva sociedad, Fundación Friedrich Ebet, [on line], № 217, septiembre-octubre de 2008, [consultado el 07 de septiembre de 2011], pp.155-171, p.162, "Disponible en la World Wide Web: http://www.nuso.org/upload/articulos/3554_1.pdf". 
En segundo término, el gobierno de Lula también intentó acelerar el crecimiento económico, pero situando al Estado como un actor central en dicho proceso. Dentro de su estrategia destacó el Programa de Aceleración del Crecimiento (PAC). Este programa, a pesar de sus tímidos resultados iniciales, reinstaló el concepto de la responsabilidad del Estado como inductor del crecimiento "al priorizar sectores y diagnosticar cuellos de botella para el crecimiento a largo plazo, con previsión de inversiones, estatales o privadas, incentivos fiscales y crédito a través de órganos oficiales, como Banco Nacional de Desarrollo Económico y Social (BNDES), Caja Económica Federal y Banco de Brasil”10.

Finalmente, Da Silva se esforzó por mejorar el sistema de protección social. En efecto, si bien su gobierno mantuvo el esquema de focalización de los programas de bienestar social -una estrategia iniciada por Cardoso que podría catalogarse de exitosa, si se consideran los drásticos recortes que están llevando a cabo los gobiernos europeos a sus generosos programas de cobertura universal- Lula amplió la cobertura de estos y reordenó administrativamente su matriz. En términos de cobertura, con Lula "la protección se amplió y se extendió a todas las familias ubicadas debajo de la línea de pobreza, en lugar de incluir solo a aquellas con características especiales. Así, las familias beneficiadas por los planes de transferencia de renta pasaron de 5,6 millones a 11,2 millones"11. Por su parte, en cuanto a la reorganización administrativa, Lula dispuso en 2004 la instalación del Ministerio del Desarrollo Social y Combate al Hambre (MDS) mediante el cual se intensificó la intersectorialidad de las políticas sociales y se creó el programa Bolsa Familia, el cual centralizó los diferentes programas de transferencia de renta existentes a la época. Esta centralización de los programas sociales no solo significó una reducción de los costos de administración de los mismos, sino que además, como ha sido reconocido, significó un avance desde el punto de vista de los derechos humanos, ya que "la centralización en un único programa evita la fragmentación y permite mayor claridad en relación a los órganos públicos responsables de su implementación. En otros términos, la centralización facilita el enfoque para solicitar el Programa, medida imprescindible para que los grupos sociales más vulnerables tengan acceso"12.

10 Dutra Fonseca, Pedro Cezar; Moreira Cunha, André; Da Silva Bichara, Julimar, Brasil antes y después de la crisis: ¿vuelve el desarrollismo?, documento de la XII Reunión de Economía Mundial - Santiago de Compostela, 26-28 de mayo, 2010, [on line], [consultado el 25 de agosto de 2011], p. 4, "Disponible en la World Wide Web: http://www.usc.es/congresos/xiirem/pdf/27.pdf".

11 Sallum Jr., Brasilio, La especificidad del gobierno de Lula. Hegemonía liberal, desarrollismo y populismo /en/ Revista Nueva Sociedad, Fundación Friedrich Ebert, [on line], № 217, septiembre-octubre de 2008, [consultado el 07 de septiembre de 2011], 155-171, p.165. "Disponible en la World Wide Web: http://www.nuso.org/upload/articulos/3554_1.pdf".

12 Zimmermann, Clóvis Roberto, Los programas sociales desde la óptica de los derechos humanos: el caso del bolsa familia del gobierno Lula en Brasil /en/ Revista Internacional de Dereitos Humanos, São Paulo, Sur-Red Universitaria de Derechos Humanos, [on line], № 4, vol. 3, junio 2006, [consultado el 08 de septiembre de 2011], 146-163, p. 154, " Disponible en la World Wide Web: http://www.scielo. br/pdf/sur/v3n4/es_08.pdf". 
Los resultados de la política económica del gobierno de Lula da Silva son evidentemente positivos. El crecimiento promedio del PIB de Brasil durante el grueso de su mandato fue más del doble del promedio del cuatrienio anterior $(3,6 \%$ anual entre 2003 y 2009, frente a 1,7\% entre 1998 y 2002) ${ }^{13}$. En 2010 la tasa de crecimiento llegaría a la cifra récord de 7,5 por ciento, registrando la mayor expansión de la economía brasileña en 24 años ${ }^{14}$. Adicionalmente, las políticas sociales ejecutadas lograron que varios millones de brasileños superaran la línea de la pobreza y que la brecha en la distribución de la riqueza se acortara durante su mandato.

Como hemos visto, las políticas económicas de los gobiernos brasileños, desde 1994 a la fecha, evolucionaron desde un modelo neoliberal absoluto hacia un modelo de mercado con mayor participación del Estado y amplias políticas de bienestar social. Esto ha significado que Brasil, a pesar de los tremendos desafíos pendientes, pueda contar actualmente con una política económica que combina adecuadamente estabilidad monetaria, crecimiento económico y mayores niveles de equidad.

\section{Una estrategia activa para el comercio regional y global}

Una de las principales características de la globalización económica es la intensificación del comercio internacional. Este fenómeno se traduce, entre otras cosas, en una mayor competencia entre las economías nacionales, que requieren formar alianzas y proyectos de asociación que les permitan negociar en mejores términos y ser más competitivas dentro de los mercados globales.

Es en este contexto que, a principios de los 90, Brasil inicia un profundo proceso de integración comercial con su principal competidor en América del Sur, Argentina. La necesidad de cooperación para ser más competitivos fue más fuerte que la larga historia de conflictos y tensiones entre ambas naciones, dando origen en 1991 al Mercado Común del Sur (MERCOSUR). A dicha unión subregional se sumarían los países limítrofes de ambos, Uruguay y Paraguay.

En línea con la tendencia del Regionalismo Abierto que experimentaba América Latina durante esos años, el primer objetivo de la alianza fue conformar una zona de libre comercio para luego avanzar hacia una unión aduanera. Esto significó, entre otras cosas, eliminar buena parte de los aranceles recíprocos y adoptar un arancel ex-

13 Infolatam, Brasil crecerá un 5,7 por ciento promedio al año hasta el 2014 según un estudio oficial, Brasilia, [on line], 10 de agosto de 2010, [consultado el 12 de septiembre de 2011], "Disponible en la World Wide Web: http://www.infolatam.com/2010/08/11/brasil-crecimiento-economico/".

14 El Mundo.es, La economía de Brasil creció un 7,5\% en 2010, su mayor expansión en 24 años, Río de Janeiro, [on line], 04 de marzo de 2011, [consultado el 25 de septiembre de 2011], "Disponible en la World Wide Web: http://www.elmundo.es/america/2011/03/03/brasil/1299163730.html". 
terno y una política comercial común. El objetivo final, teniendo en mente el modelo de integración por etapas implementado por la Unión Europea, era la creación de un mercado único y la coordinación de las políticas macroeconómicas de los Estados parte.

Al día de hoy, MERCOSUR no ha logrado alcanzar los objetivos trazados en la década del 90. De hecho, actualmente es considerada una unión aduanera imperfecta por la serie de "perforaciones" de sus políticas arancelarias. Adicionalmente, los niveles de comercio dentro del bloque han experimentado importantes reducciones. Esta ralentización del comercio regional -fenómeno también observado en otros pactos sudamericanos, como en la Comunidad Andina (CAN) - ha sido atribuida por algunos autores al impacto negativo de las crisis financieras de otras latitudes, a la insuficiente voluntad política de sus miembros para buscar mecanismos más profundos de integración macroeconómica y a la incompleta evolución institucional de los mismos acuerdos ${ }^{15}$. A pesar de todo, el MERCOSUR logró instalar el discurso de la integración en la región. Y en ese sentido, dentro de sus logros, se encontraría el haber sentado las bases para proyectos de integración más ambiciosos como la Unión de Naciones Sudamericanas (UNASUR).

Pasando al ámbito extrarregional, durante los últimos años Brasil ha comenzado a ser considerado un actor político y económico de primer orden. Los analistas suelen calificar a Brasil como una potencia intermedia, es decir, aquel país que cuenta con las condiciones para convertirse en potencia mundial. Este ascenso y reconocimiento internacional se debe en gran medida a la compleja y diversificada red de relaciones comerciales que ha logrado desarrollar la política exterior de Itamaraty, la cual revisaremos a continuación.

Primero que todo, Brasil ha procurado mantener un buen nivel de comercio bilateral con Estados Unidos. Asimismo, ha sido persistente en su objetivo de suscribir, en el marco del MERCOSUR, un tratado de libre comercio con la Unión Europea (en mayo de 2010 se reanudaron las negociaciones luego de seis años de estancamiento). De este modo, Brasil ha asegurado contar con una sólida base de relaciones económicas con el mundo desarrollado.

No obstante, lo cierto es que durante la última década y siguiendo la tendencia regional, Brasil ha orientado su política económica principalmente hacia el sur, en particular hacia el Asia. Esta nueva orientación ha respondido a múltiples factores y a continuación analizaremos dos de los principales.

15 Di Filippo, Armando, América Latina: Integración Regional en la Era Global, [on line], [consultado el 14 de septiembre de 2011], p.15, "Disponible en la World Wide Web: http://www.difilippo.cl/articulos_RRII.php". 
En primer factor determinante ha sido el gran dinamismo y el sostenido crecimiento que han experimentado las economías de China e India. La necesidad de estas naciones de mantener altísimos ritmos de expansión y alimentar a una numerosa población, ha significado el auge del mercado de productos básicos de América Latina. Este hecho ya lo destacaba la Comisión Económica para América Latina y el Caribe (CEPAL) cuando en 2005 señalaba "el notable dinamismo que viene registrando China y la India, que en 2005 crecieron a tasas superiores al $9 \%$ y $8 \%$, respectivamente, ha impulsado una mejora en los términos del intercambio de los países exportadores de productos básicos, que a su vez ha permitido a las economías emergentes mantener altas tasas de expansión. Por consiguiente, los precios promedio de los productos básicos exportados por América Latina siguieron elevándose a tasas superiores al $13 \% " 16$.

El auge de estos productos de primera necesidad ha significado que después de 50 años, China haya reemplazado a Estados Unidos como el principal socio comercial de Brasil. La economía brasileña se ha logrado posicionar como la fuente clave de recursos minerales, petróleo y soya del mercado chino, lo que ha llevado a que durante la última década el comercio entre China y Brasil se disparara desde apenas US\$2.300 millones en 2000 a US\$36.100 millones en $2009^{17}$.

Un segundo factor que ha llevado a que Brasil privilegie la integración económica sur-sur ha sido la necesidad de entablar una alianza con los principales países emergentes. En esto la complementariedad de las economías ha sido importante, pero el elemento detonante ha estado en la posibilidad de mejorar los términos de intercambio con los países desarrollados del norte. Esta estrategia quedó de manifiesto tanto en el rechazo de Brasil a las negociaciones de la Ronda de Doha, como en la creación del Foro multilateral IBSA.

En efecto, durante las negociaciones de 2003 de la OMC, Ilevadas a cabo en Doha y concluidas en Cancún, Brasil manifestó su firme oposición -junto a China y varios países del hemisferio Sur- a la última oferta del bloque de Estados desarrollados. La oposición se fundamentó en la defensa que Estados Unidos y la Unión Europea hacían de las subvenciones agrarias. El resultado no solo fue el fracaso de las negociaciones, sino también la articulación de un nuevo referente de países que incluye a las potencias medias y Estados emergentes, el Ilamado G-20 ${ }^{18}$.

16 CEPAL, Estudio Económico de América Latina y el Caribe 2005-2006 (LC/G. 2314-P/E), Publicación de las Naciones Unidas, Santiago de Chile, 2006, p.14.

17 The Wall Street Journal, Cumbre Brasil-China; los BRIC se ponen en acción, 14 de abril de 2010, [on line], [consultado el 17 de agosto de 2011], "Disponible en la World Wide Web: www.estrategiaynegocios.net/mundo/Default.aspx?option=9392".

18 Grabendorff, Wolf, Brasil: de coloso regional a potencia global /en/ Revista Nueva Sociedad, Fundación Friedrich Ebert, [on line], № 226, marzo-abril de 2010, [consultado el 14 de septiembre de 2011], 
Por su parte, la articulación ese mismo año de la alianza trilateral entre India, Brasil y Sudáfrica (IBSA) fue una de las consecuencias del fracaso de Doha. Sin embargo, los propósitos del Foro fueron más allá de la promoción del comercio entre sus respectivas economías. Entre otras cosas, el IBSA se fijó como objetivo proteger los intereses económicos de los países en desarrollo ante las fluctuaciones globales de los precios de las materias primas. Esto quedó de manifiesto en el punto 15 de la Declaración de Brasilia en que los firmantes señalaron: "The Foreign Ministers noted with concern the increased economic vulnerability of developing countries to fluctuations in global prices of commodities. They affirmed the importance of a predictable, rule-based, and transparent international trading system, to enable the developing countries to maximise their development, through gains from enhanced exports of goods and services of their competitive advantage ${ }^{\prime 19}$. Y la misma Declaración permite sostener que el objetivo del foro es aún más amplio y consiste en definitiva en perseguir un reparto más equitativo de los beneficios de la globalización. Al respecto, su cláusula $13^{\text {a }}$ es clara al señalar: "While welcoming the expansion of economic growth, employment, and social development, and the accompanying rise in standards of living, in several developing countries as a result of freer movements of trade, capital, and technology, the Foreign Ministers of Brazil, India and South Africa expressed their concern that large parts of the world have not benefited from globalization. They agreed that globalization must become a positive force for change for all peoples, and must benefit the largest number of countries. In this context, they affirmed their commitment to pursuing policies, programmes and initiatives in different international forums, to make the diverse processes of globalization inclusive, integrative, humane, and equitable" ${ }^{\prime 20}$.

Como hemos visto, Brasil ha carecido de inhibiciones en su intención de jugar un rol estelar en las esferas del comercio internacional. Por una parte, Brasil ha sabido aprovechar las ventajas del fenómeno globalizador, convirtiéndose en un socio fundamental de los principales mercados globales. Pero, al mismo tiempo, su adaptación a la actual realidad económica no ha sido pasiva. A través de una audaz política exterior, consciente de la necesidad de promover una mejor distribución de los recursos, Brasil ha diseñado una estratégica política de alianzas, que le ha permitido levantar la voz de los países emergentes y hacer frente a las exigencias de las principales economías desarrolladas.

pp.158-171, p.163, “Disponible en la World Wide Web: http://www.nuso.org/upload/articulos/3691_1. pdf".

19 IBSA, Brasilia Declaration, [on line], [consultado el 14 de septiembre de 2011] "Disponible en la World Wide Web: www.ibsa.nic.in/brasil_declaration.htm".

20 IBSA, Brasilia Declaration, [on line], [consultado el 14 de septiembre de 2011], "Disponible en la World Wide Web: www.ibsa.nic.in/brasil_declaration.htm". 


\section{Bibliografía}

Brasil.gov.br, [on line], [consultado el 27 de abril de 2012], "Disponible en la World Wide Web: http://www.brasil.gov.br/sobre/brasil-1/brasil-en-numeros/demografia".

CEPAL, Estudio Económico de América Latina y el Caribe 2005-2006 (LC/G. 2314P/E), Publicación de las Naciones Unidas, Santiago de Chile, 2006, p.14.

CEPAL, Transformación productiva con equidad. La tarea prioritaria del desarrollo de América Latina y el Caribe en los años noventa (LC/G. 1601-P), Publicación de las Naciones Unidas, Santiago de Chile, 1990, Nº33.

Dı FILIPPO, Armando, América Latina: Integración Regional en la Era Global, [on line], [consultado el 14 de septiembre de 2011], p.15, "Disponible en la World Wide Web: http://www.difilippo.cl/articulos_RRII.php".

Dı FILIPPO, Armando, Economía Política Global, [on line], 2011, [consultado el 14 de septiembre de 2011], p.178, "Disponible en la World Wide Web: http://www. difilippo.cl/descargar_ap.php?id=178".

Dutra Fonseca, Pedro Cezar; Moreira Cunha, André; Da Silva Bichara, Julimar, Brasil antes y después de la crisis: ¿vuelve el desarrollismo?, documento de la XII Reunión de Economía Mundial - Santiago de Compostela, 26-28 de Mayo, 2010, [on line], [consultado el 25 de agosto de 2011], p.4, "Disponible en la World Wide Web: http://www.usc.es/congresos/xiirem/pdf/27.pdf".

El Mundo.es, La economía de Brasil creció un 7,5\% en 2010, su mayor expansión en 24 años, Río de Janeiro, [on line], 04 de marzo de 2011, [consultado el 25 de septiembre de 2011], "Disponible en la World Wide Web: http://www.elmundo. es/america/2011/03/03/brasil/1299163730.html".

GrabendorfF, Wolf, Brasil: de coloso regional a potencia global /en/ Revista Nueva Sociedad, Fundación Friedrich Ebert, [on line], № 226, marzo-abril de 2010, [consultado el 14 de septiembre de 2011], 158-171, p.163, "Disponible en la World Wide Web: http://www.nuso.org/upload/articulos/3691_1.pdf".

IBSA, Brasilia Declaration, [on line], [consultado el 14 de septiembre de 2011], "Disponible en la World Wide Web: www.ibsa.nic.in/brasil_declaration.htm".

Infolatam, Brasil crecerá un 5,7 por ciento promedio al año hasta el 2014 según un estudio oficial, Brasilia, [on line], 10 de agosto de 2010, [consultado el 12 de septiembre de 2011], "Disponible en la World Wide Web: http://www.infolatam. com/2010/08/11/brasil-crecimiento-economico/".

Informe sobre Desarrollo Humano 2010, Programa de las Naciones Unidas para el Desarrollo (PNUD). [on line], [consultado el 27 de abril de 2012], "Disponible en la World Wide Web: http://hdr.undp.org/en/media/HDR_2010_ES_Complete_reprint.pdf". 
Saltum JR., Brasilio, La especificidad del gobierno de Lula. Hegemonía liberal, desarrollismo y populismo / en / Revista Nueva sociedad, Fundación Friedrich Ebet, [on line], № 217, septiembre-octubre de 2008, [consultado el 07 de septiembre de 2011], pp.155-171, "Disponible en la World Wide Web: http://www.nuso. org/upload/articulos/3554_1.pdf".

The Wall Street Journal, Cumbre Brasil-China; los BRIC se ponen en acción, 14 de abril de 2010, [on line], [consultado el 17 de agosto de 2011], "Disponible en la World Wide Web: www.estrategiaynegocios.net/mundo/Default. aspx?option=9392".

Zimmermann, Clóvis Roberto, Los programas sociales desde la óptica de los derechos humanos: el caso del bolsa familia del gobierno Lula en Brasil /en/ Revista Internacional de Dereitos Humanos, São Paulo, Sur-Red Universitaria de Derechos Humanos, [on line], $\mathrm{N}^{\circ} 4$, vol. 3, junio 2006, [consultado el 08 de septiembre de 2011], 146-163, p. 154, "Disponible en la World Wide Web: http://www. scielo.br/pdf/sur/v3n4/es_08.pdf".

World Economic Outlook Database, April 2012. [on line], [consultado el 27 de abril de 2012], "Disponible en la World Wide Web: http://www.imf.org/external/ pubs/ft/weo/2012/01/weodata/index.aspx". 